\title{
Octet Baryon Charge Radii, Chiral Symmetry and Decuplet Intermediate States
}

\author{
S.J. Puglia ${ }^{a}$ \\ M.J. Ramsey-Musolf ${ }^{a, b}$ \\ Shi-Lin $\mathrm{Zhu}^{a}$ \\ a Department of Physics, University of Connecticut, Storrs, CT 06269 USA \\ ${ }^{b}$ Theory Group, Thomas Jefferson National Accelerator Facility, Newport News, VA 23606
}

\begin{abstract}
We compute the octet baryon charge radii to $\mathcal{O}\left(1 / \Lambda_{\chi}^{2} M_{N}\right)$ in heavy baryon chiral perturbation theory $(\mathrm{HB} \chi \mathrm{PT})$. We examine the effect of including the decuplet of spin- $\frac{3}{2}$ baryons explicitly. We find that it does not improve the level of agreement between the $\mathrm{HB} \chi \mathrm{PT}$ and experimental values for the $\Sigma^{-}$ charge radius.
\end{abstract}

The study of electromagnetic (EM) form factors is an important tool used in developing a theoretical understanding of the internal structure of hadrons. In particular, the application of chiral perturbation theory $(\chi \mathrm{PT})$ to this study allows one to disentangle the long- and short-range QCD dynamics governing the behavior of form factors at low momentum transfers. The long-range effects appear in the guise of non-analytic loop contributions while the short-range dynamics are subsumed into phenomenologically determined low-energy constants (LEC's).

An important issue in the viability of this program is the convergence of the chiral expansion. An important consideration in obtaining proper covergence is the treatment of the large baryon mass. In many cases, one obtains adequate convergence by employing a non-relativistic version of $\chi \mathrm{PT}$, involving a second expansion in inverse powers of the baryon mass. In addition, it has been found that for some observables, explicit inclusion of the decuplet of spin- $\frac{3}{2}$ baryons is necessary in order to achieve the expected convergence behavior. For octet of axial currents [6], for example, the contribution of the decuplet is comparable to that of the octet and opposite in sign, leading to sizeable cancellations. In the case of the octet baryon magnetic moments, this convergence has been a subject of controversy [2] [10]. Recently, however, it was shown that inclusion of decuplet intermediate states - together with the full set of $1 / M_{N}$ corrections - produces the expected behavior of the expansion [5].

In contrast, the behavior of the corresponding expansion for the baryon octet charge radii has not been extensively studied. Existing calculations have worked only to leading order in $1 / \Lambda_{\chi}$, where $\Lambda_{\chi}=4 \pi F_{\pi} \sim 1 \mathrm{GeV}$ is the scale of chiral symmetry breaking [8] [9]. The recent SELEX measurement of the $\Sigma^{-}$charge radius [4] makes further investigation of the chiral 
convergence for the octet radii timely. In this paper, we address this issue by computing the radii through sub-leading order. As in the case of the magnetic moments, we consider the impact of explicit inclusion of decuplet intermediate states as well as $1 / M_{N}$ corrections. We find that at order $1 /$ Heavy $^{3}$, where "Heavy" denotes either $\Lambda_{\chi}$ or $M_{N}$, the chiral expansion for the charge radii does not appear to be under control. Moreover, attaining further progress is hampered by the lack of available data. Although a computation of the next order nonanalytic contributions feasible, the new LEC's arising at this order could not be determined from experiment. Thus, when baryons are treated in a non-relativistic framework, a chiral analysis of baryon charge radii is viable only for the nucleon isovector radius in the context of $\mathrm{SU}(2)$ ).

$\chi \mathrm{PT}$ is an effective field theory of the strong interactions which exploits the approximate chiral symmetry of the QCD Lagrangian. The spontaneous breakdown of this symmetry gives rise to eight (nearly) massless Goldstone bosons. These are identified with the octet of pseudoscalar mesons. In the meson sector a well-defined low-energy expansion in terms of the meson 4-momentum can be constructed to describe the dynamics. Here low-energy is defined relative to the scale of chiral symmetry breaking $\Lambda_{\chi}=4 \pi F_{\pi} \approx 1 \mathrm{GeV}$, where $F_{\pi}$ is the pion decay constant. The baryons can be included in a consistent manner as shown in Ref. [6] The non-relativistic version of $\chi \mathrm{PT}$ including the baryons - heavy baryon chiral perturbation theory $(\mathrm{HB} \chi \mathrm{PT})$ - has many simplifying features. A review of these features can be found in Ref. [3]. $\mathrm{HB} \chi \mathrm{PT}$ has proven useful in the calculation of low-energy observables such as baryon masses, Compton scattering amplitudes, nucleon polarizabilities, sigma terms, axial cuurent, and hyperon decays [四]. The important point for the present study is that $\mathrm{HB} \chi \mathrm{PT}$ introduces a second expansion scale $M_{N}$ commensurate with $\Lambda_{\chi}$. Thus, one should include all contributions to a given order in 1/Heavy, where "Heavy" denotes either $\Lambda_{\chi}$ or $M_{N}$. In Ref. [10] three-flavor HB $\chi \mathrm{PT}$ was used to calculate the EM form fatcors for the octet. The analysis there was carried out to $\mathcal{O}\left(1 / \mathrm{Heavy}^{2}\right)$ and did not include the decuplet as an explicit degree of freedom. Our approach differs from theirs in two respects: 1.) Inclusion of the decuplet, 2.) inclusion of $1 /\left(M_{N}\right)$ corrections.

The formalism of $\mathrm{HB} \chi \mathrm{PT}$ is by now well known and we refer the reader to Ref. [5] for our conventions and notation. As in Ref. [10] we define the charge radius by

$$
\left\langle r_{E}^{2}\right\rangle=\left.\frac{1}{N} \frac{d G_{E}\left(q^{2}\right)}{d q^{2}}\right|_{q^{2}=0}
$$

where $G_{E}$ is the Sachs electric form factor and $N$ is a normalization constant. The tree-level contributions to the charge radii are $\mathcal{O}\left(1 / \Lambda_{\chi}^{2}\right)$ in the chiral expansion and are generated by the Lagrangian [7]

$$
\mathcal{L}_{2}=-\frac{e}{\Lambda_{\chi}^{2}}\left\{c_{+} \operatorname{Tr}\left(\bar{B}_{v}\left\{Q, B_{v}\right\}\right)+c_{-} \operatorname{Tr}\left(\bar{B}_{v}\left[Q, B_{v}\right]\right)\right\} v_{\mu} \partial_{\nu} F^{\mu \nu}
$$

\footnotetext{
${ }^{1}$ For an analsis of nucleon EM form factors in relativistic baryon $\chi \mathrm{PT}$, see Ref. 11].
} 
There is an additional tree-level contribution at $1 /(\text { Heavy })^{2}$ that arises from the $1 / M_{N}$ correction of the kinetic term of the lowest order Lagrangian and is given by

$$
\mathcal{L}_{\frac{1}{M_{N}{ }^{2}}}=-\frac{1}{4 M_{N}^{2}} \operatorname{Tr}\left(\bar{B}_{v}\left[\not D^{\perp},\left[v \cdot D,\left[\not D^{\perp}, B_{v}\right]\right]\right]\right\},
$$

where $D_{\mu}^{\perp}=D_{\mu}-v_{\mu} v \cdot D$.

The relevant one-loop graphs which arise at order $\mathcal{O}\left(1 / \Lambda_{\chi}^{2}\right)$ are shown in Fig. 1 (b-f), where the vertices arise from the Lagrangian: [12]

$$
\begin{aligned}
\mathcal{L}_{0}= & i \operatorname{Tr}\left(\bar{B}_{v} v \cdot D B_{v}\right)+2 D \operatorname{Tr}\left(\bar{B}_{v} S_{v}^{\mu}\left\{A_{\mu}, B_{v}\right\}\right)+2 F \operatorname{Tr}\left(\bar{B}_{v} S_{v}^{\mu}\left[A_{\mu}, B_{v}\right]\right) \\
& -i \bar{T}_{v}^{\mu}(v \cdot \mathcal{D}) T_{v \mu}+\delta \bar{T}_{v}^{\mu} T_{v \mu}+\mathcal{C}\left(\bar{T}_{v}^{\mu} A_{\mu} B_{v}+\bar{B}_{v} A_{\mu} T_{v \mu}\right)+2 \mathcal{H} \bar{T}_{v}^{\mu} S_{v}^{\nu} A_{\nu} T_{v \mu} \\
& +\frac{f_{\pi}^{2}}{4} \operatorname{Tr}\left(\left(D^{\mu} \Sigma\right)^{\dagger} D_{\mu} \Sigma\right) .
\end{aligned}
$$

In the following we take $D=.75, F=.50$ and $\mathcal{C}=-1.5$

The loop corrections to the charge radius at $1 /(\text { Heavy })^{2}$ are represented by the graphs (c-g) in Fig. 1. The contributions to the charge radii from (d),(e) and (g) vanish indentically since these graphs have no $q^{2}$ dependence at this order in the chiral expansion. With the inclusion of the counterterm and the $1 / M_{N}{ }^{2}$ correction, the charge radii have the form:

$$
\left\langle r_{B}^{2}\right\rangle=\frac{6 N}{\Lambda_{\chi}^{2}}\left\{\alpha_{B}-\sum_{X=\pi, K}\left(\beta_{B}^{(X)} \ln \frac{m_{X}^{2}}{\mu^{2}}+\beta_{B}^{\prime(X)} F\left(m_{X}, \delta, \mu\right)\right)\right\}+\frac{3 \tilde{\alpha}_{B}}{2 \Lambda_{\chi} M_{N}}-\frac{3 Q_{B}}{4 M_{N}^{2}},
$$

Here $N$ is a normalization factor which is equal to the charge of the baryon if it is charged or 1 otherwise and $Q_{B}$ is the charge of the baryon. The coefficients $\alpha_{B}$ are linear combinations of the counterterm couplings $c_{ \pm}$from Eq.(2), while the $\beta_{B}^{(X)}$ and $\beta_{B}^{\prime(X)}$ are products of the meson charge and Clebsch-Gordon coefficients arising from the one-loop graphs in Fig. 1. The second to last term is the so called "Foldy"-term [11] and arises from the definition of the $G_{E}$. This term depends on two magnetic couplings which we call $b_{ \pm}$and are contained in the $\tilde{\alpha}_{B}$ 's [10, 11]. The last term in Eq.(50) is the relativistic correction from Eq.(3).

We note that the coefficients, $\beta_{B}^{\prime(X)}$, for the graph containing the decuplet have not been computed before. All of these coefficients are listed in the Appendix. The analytic structure for this graph is given by:

$$
F(m, \delta, \mu)=\ln \frac{m^{2}}{\mu^{2}}+2 \delta \begin{cases}\frac{1}{\sqrt{m^{2}-\delta^{2}}}\left(\frac{\pi}{2}-\arctan \left[\frac{\delta}{\sqrt{m^{2}-\delta^{2}}}\right]\right) & m \geq \delta \\ -\frac{1}{\sqrt{\delta^{2}-m^{2}}} \ln \left[\frac{\delta+\sqrt{\delta^{2}-m^{2}}}{m}\right] & m<\delta,\end{cases}
$$

where $\delta$ is the octet-decuplet mass splitting and $\mu$ is the renormalization scale.

The contributions at $\mathcal{O}\left(1 /\right.$ Heavy $\left.^{3}\right)$ are all proportional to $1 / \Lambda_{\chi}^{2} M_{N}$. There are no terms proprtional to $1 / \Lambda_{\chi}^{3}$ with the correct spacetime structure and terms proportional to $1 / \Lambda_{\chi} M_{N}{ }^{2}$ or $1 / M_{N}{ }^{3}$ will vanish at $q^{2}=0$. The terms proportional to $1 / \Lambda_{\chi}^{2} M_{N}$ are of two types. First 
the graphs in Fig. 1 can be expanded as functions of $q^{2}$ and it is found that they contain contributions proportional to $q^{2} / 2 M_{N}$. The graphs (c), (d), (f) and (g) are of this type and will contribute to the charge radius at this order. There are also graphs that contain an explicit factor of $1 / M_{N}$ which arises for the heavy baryon expansion. These are the graphs shown in Fig. 2, which contain vertices generated from the Lagragian [10]

$$
\begin{aligned}
\mathcal{L}_{M_{N}}= & \frac{1}{2 M_{N}} \operatorname{Tr}\left(\bar{B}[v \cdot D,[v \cdot D, B]]-\bar{B}\left[D^{\mu},\left[D_{\mu}, B\right]\right]\right) \\
& -\frac{i D}{2 M_{N}} \operatorname{Tr}\left(\bar{B} S_{\mu}\left[D^{\mu},\{v \cdot A, B\}\right]+\bar{B} S_{\mu}\left\{v \cdot A,\left[D^{\mu}, B\right]\right\}\right) \\
& -\frac{i F}{2 M_{N}} \operatorname{Tr}\left(\bar{B} S_{\mu}\left[D^{\mu},[v \cdot A, B]\right]+\left\langle\bar{B} S_{\mu}\left[v \cdot A,\left[D^{\mu}, B\right]\right]\right)\right. \\
& +\frac{1}{2 M_{N}} \bar{T}_{v}^{\mu}\left(\mathcal{D}^{\alpha} \mathcal{D}_{\alpha}-v \cdot \mathcal{D} v \cdot \mathcal{D}\right) T_{v \mu} .
\end{aligned}
$$

Although there are thirteen graphs which appear at $\mathcal{O}\left(1 / \mathrm{Heavy}^{3}\right)$, the contributions from several vanish. The amplitude of Fig 1. (e) again has no $q^{2}$ dependence. The $1 / M_{N}$ contribution from Fig 1. (d) combines with those of Fig 2. (c) and (d) to sum to zero. A similar cancellation takes place between the $1 / M_{N}$ correction to Fig. 1 (g) and Fig. 2 (e) and (f). The graphs in Fig. $2(\mathrm{~g})$ and $(\mathrm{h})$ vanish at this order. Thus, only those in Fig. 1 (c) and (f) and Fig. 2 (a) and (b) actually end up contributing to the charge radius. Taking into account these contributions the charge radius now has the form:

$$
\begin{aligned}
\left\langle r_{B}^{2}\right\rangle= & \frac{6 N}{\Lambda_{\chi}^{2}}\left\{\alpha_{B}-\sum_{X=\pi, K}\left(\beta_{B}^{(X)}\left(\ln \frac{m_{X}^{2}}{\mu^{2}}+\frac{23 \pi m_{X}}{24 M_{N}}\right)+\beta_{B}^{\prime(X)}\left(F\left(m_{X}, \delta, \mu\right)-\frac{2}{M_{N}} G\left(m_{X}, \delta, \mu\right)\right)\right)\right\} \\
& +\frac{3 \tilde{\alpha}_{B}}{2 \Lambda_{\chi} M_{N}}-\frac{3 Q_{B}}{4 M_{N}{ }^{2}}
\end{aligned}
$$

where

$$
G(m, \delta, \mu)=\delta \ln \frac{m^{2}}{\mu^{2}}+\left(2 \delta^{2}-m^{2}\right) \begin{cases}\frac{1}{\sqrt{m^{2}-\delta^{2}}}\left(\frac{\pi}{2}-\arctan \left[\frac{\delta}{\sqrt{m^{2}-\delta^{2}}}\right]\right) & m \geq \delta \\ \frac{1}{\sqrt{\delta^{2}-m^{2}}} \ln \left[\frac{\delta+\sqrt{\delta^{2}-m^{2}}}{m}\right] & m<\delta .\end{cases}
$$

We now turn to the determination of the LEC's $c_{ \pm}$. First, we need values for the magnetic couplings $b_{ \pm}$which appear in the Foldy term. These are obtained from a fit of the octet anomalous magnetic moments [5] and are given in Table I for the cases considered below. With these, we use the measured values of the nucleon charge radii to determine $c_{ \pm}$. We consider two cases: 1.) octet intermediate states only $(\mathrm{O}), 2$.$) both octet and decuplet$ intermediate states included $(\mathrm{O}+\mathrm{D})$. The values for the counterterm couplings and charge radii in each case are shown in Tables II and III respectively.

We see that the effect of the decuplet is large and, except for $\Xi^{0}$ and $\Lambda$, tends to increase the value of $\left\langle r_{B}^{2}\right\rangle$. In the case of the $\Sigma^{-}$at $\mathcal{O}\left(1 / \Lambda_{\chi}^{2}\right)$ both the $\mathrm{O}$ and $\mathrm{O}+\mathrm{D}$ calculations 
produce a value for the charge radius that is much larger than the SELEX measurement of $\left\langle r_{\Sigma^{-}}^{2}\right\rangle=0.60 \pm 0.08$. The $1 / M_{N}$ corrections tend to reduce this result, with the $\mathcal{O}\left(1 / \Lambda_{\chi}^{2} M_{N}\right)$ octet only value being very close to the measured value. Here again, however, the addition of the decuplet increases the predicted value away from the measured one. We also note the fluctuations in sign for the calculated values for $\Xi^{0}, \Lambda$ and the $\Sigma^{0} \Lambda$ transition moment.

The effect of including both the decuplet and the $1 / M_{N}$ corrections on the LEC's is also dramatic. While in all cases $c_{ \pm}$are of natural size, they change considerably, both in sign and in magnitude, between orders and with the addition of the decuplet. No clear pattern emerges and the values do not appear to be converging.

We contrast the above behavior to the magnetic moment case as analyzed in Ref. [5]. There, inclusion of both the decuplet and the $1 /\left(\right.$ Heavy $\left.^{3}\right)$ terms played a essential role obtaining the proper convergence behavior of the chiral expansion. In that case, it was possible to work two orders beyond tree-level since there is ample data to fit higher order counterterms and no two-loop contributions. For the charge radii, however, the lowest order loop and tree-level contributions arise at the same order. Moreover, the exists considerably less data for the charge radii than for the magnetic moments. Consequently, it is impossible to analyze the radii beyond sub-leading order in $\mathrm{HB} \chi \mathrm{PT}$ without introducing strong model assumptions. Indeed, at next-to-next-to-leading order, one must compute the full set of two-loop contributions and include a plethora of new LEC's. The lack of sufficient data would require the use of a model to determine the latter.

In short, we conclude that the heavy baryon chiral expansion of the baryon octet charge radii is not yet under control. One must include the octet radii in a growing list of observables for which the convergence of the non-relativistic chiral expansion appears problematic at best (see, e.g., Refs. 13 15.) In this regard, a reanalysis of the octet magnetic moments and charge radii may prove more successful when a relativistic framework is adopted [11]. It remains to be seen whether such a framework sufficiently reduces the number of LEC's incurred in the heavy baryon expansion and circumvents the need to work beyond subleading order in analyzing octet EM moments. 


\section{REFERENCES}

[1] See. e.g., V. Bernard, N. Kaiser, U-G. Meißner, Z. Phys C60 (1993) 111; B.R. Holstein, Comments Nucl. Part. Phys. 20, 301 (1992); V. Bernard, N. Kaiser, J. Kambor, U-G. Meißner, Nucl. Phys. B388, (1992) 301; B. Borasay, Eur. Phys. J C8 (1999) 121; J. Bijnens, H.Sonoda, M.B. Wise, Nucl. Phys B261, (1999) 185.

[2] E. Jenkins, M. Luke, A.V. Manohar and M.Savage, Phys. Lett. B 302 (1993) 482; B 388 (1996) (E); Loyal Durand and Phuoc Ha, Phys. Rev. D58 (1998) 13010; M. N. Butler, M.J. Savage, R.P. Springer, Phys. Rev. D (1994) 3459.

[3] E. Jenkins and A.V. Manohar, Baryon chiral perturbation theory, in: Proc. Workshop on effective field theories of the standard model, ed. Ulf-G. Meißner (World Scientific, Singapore, 1992).

[4] I. Eschrich on behalf of the SELEX Collaboration, Measurement of the $\Sigma^{-}$Charge Radius at SELEX in: Proceedings of the 8th Int. Conference on the Structure of Baryons (BARYONS'98), Bonn, Germany, Sept 22-26, (World Scientific, Singapore, 1998).

[5] S.J. Puglia, M. J Ramsey-Musolf, Phys. Rev. D62 (2000) 034010.

[6] E. Jenkins and A.V. Manohar, Phys. Lett. B 259 (1991) 353.

[7] M. J. Ramsey-Musolf and Hiroshi Ito, Phys. Rev. C (1997) 2066.

[8] B. Kubis, T. Hemmert, Ulf-G. Meißner, Phys. Lett. B456 (1999) 240

[9] T. Cohen, Phys.Lett. B359 (1995) 23

[10] Ulf-G. Meißner and S. Steininger, Nucl. Phys. B 499 (1997) 349.

[11] B. Kubis, Ulf-G. Meißner, hep-ph/0007056

[12] T. Hemmert, B.R. Holstein, J. Kambor, J. Phys. G24 (1998) 1831.

[13] Ulf-G. Meißner, hep-ph/9709402.

[14] T. Hemmert and Ulf-G. Meißner, Phys. Rev. Lett. 85 (2000) 14.

[15] K. B. V. Kumar, J. A. McGovern and M. C. Birse, Phys. Lett. B 479 (2000) 167. 


\section{TABLES}

\begin{tabular}{|l|r|r|r|r|}
\hline \hline \hline & & $\mathcal{O}\left(1 / \Lambda_{\chi}^{2}\right)$ & & \multicolumn{2}{|c|}{$\mathcal{O}\left(1 / \Lambda_{\chi}^{2} M_{N}\right)$} \\
\hline & $\mathrm{O}$ & $\mathrm{O}+\mathrm{D}$ & $\mathrm{O}$ & $\mathrm{O}+\mathrm{D}$ \\
\hline & & & & \\
$b_{+}$ & 2.999 & 3.61 & 1.975 & 3.104 \\
$b_{-}$ & 1.571 & 1.571 & 1.042 & 1.393 \\
& & & & \\
\hline \hline
\end{tabular}

TABLE I. Magnetic couplings appearing in the Foldy-term of the charge radius.

\begin{tabular}{|l|r|r|r|r|}
\hline \hline \hline & & $\mathcal{O}\left(1 / \Lambda_{\chi}^{2}\right)$ & \multicolumn{2}{|c|}{$\mathcal{O}\left(1 / \Lambda_{\chi}^{2} M_{N}\right)$} \\
\hline$C T$ & $\mathrm{O}$ & $\mathrm{O}+\mathrm{D}$ & $\mathrm{O}$ & $\mathrm{O}+\mathrm{D}$ \\
\hline & & & & \\
$c_{+}$ & -4.089 & -4.620 & 0.202 & 0.286 \\
$c_{-}$ & 1.410 & -0.211 & 2.888 & 1.051 \\
& & & & \\
\hline \hline
\end{tabular}

TABLE II. Couplings for leading order Charge Radius Counterterm.

\begin{tabular}{|c|c|c|c|c|}
\hline & \multicolumn{4}{|c|}{$\left\langle\overline{\left\langle r_{B}^{2}\right\rangle\left(\mathrm{fm}^{2}\right)}\right.$} \\
\hline & \multicolumn{2}{|c|}{$\mathcal{O}\left(1 / \Lambda_{\chi}^{2}\right)$} & \multicolumn{2}{|c|}{$\mathcal{O}\left(1 / \Lambda_{\chi}^{2} M_{N}\right)$} \\
\hline & $\mathrm{O}$ & $\mathrm{O}+\mathrm{D}$ & $\mathrm{O}$ & $\mathrm{O}+\mathrm{D}$ \\
\hline$p$ & 0.735 & 0.735 & 0.735 & 0.735 \\
\hline$n$ & -0.113 & -0.113 & -0.113 & -0.113 \\
\hline$\Xi^{0}$ & 0.251 & -0.001 & 0.112 & -0.122 \\
\hline$\Xi^{-}$ & 0.599 & 0.851 & 0.763 & 0.997 \\
\hline$\Sigma^{+}$ & 0.647 & 1.522 & 0.781 & 1.366 \\
\hline$\Sigma^{-}$ & 0.851 & 0.977 & 0.681 & 0.798 \\
\hline$\Lambda$ & 0.102 & -0.150 & -0.050 & -0.284 \\
\hline$\Sigma^{0} \Lambda$ & -0.021 & -0.021 & 0.074 & 0.074 \\
\hline
\end{tabular}

TABLE III. Calculated values Charge Radii from fit of Counterterms to Nucleon experimental values. 


\section{APPENDIX}

Here we tabulate the coeffcients appearing the expressions for the charge radii.

\begin{tabular}{|l|l|}
\hline & \multicolumn{1}{|c|}{$\alpha_{B}$} \\
\hline$p$ & $\frac{1}{3} c_{+}+c_{-}$ \\
$n$ & $-\frac{2}{3} c_{+}$ \\
$\Xi^{-}$ & $-\frac{1}{3} c_{+}-c_{-}$ \\
$\Xi^{0}$ & $\frac{-2}{3} c_{+}$ \\
$\Sigma^{+}$ & $\frac{1}{3} c_{+}+c_{-}$ \\
$\Sigma^{-}$ & $\frac{1}{3} c_{+}-c_{-}$ \\
$\Lambda$ & $-\frac{1}{3} c_{+}$ \\
$\Sigma^{0} \Lambda$ & $\frac{1}{\sqrt{3}} c_{+}$ \\
\hline
\end{tabular}

\begin{tabular}{|l|l|}
\hline & \multicolumn{1}{|c|}{$\tilde{\alpha}_{B}$} \\
\hline$p$ & $\frac{1}{3} b_{+}+b_{-}$ \\
$n$ & $-\frac{2}{3} b_{+}$ \\
$\Xi^{-}$ & $-\frac{1}{3} b_{+}-b_{-}$ \\
$\Xi^{0}$ & $\frac{-2}{3} b_{+}$ \\
$\Sigma^{+}$ & $\frac{1}{3} b_{+}+b_{-}$ \\
$\Sigma^{-}$ & $\frac{1}{3} b_{+}-b_{-}$ \\
$\Lambda$ & $-\frac{1}{3} b_{+}$ \\
$\Sigma^{0} \Lambda$ & $\frac{1}{\sqrt{3}} b_{+}$ \\
\hline
\end{tabular}




\begin{tabular}{|l|c|c|}
\hline & \multicolumn{2}{|c|}{$\beta_{B}^{(X)}$} \\
\hline & $\pi$ & $K$ \\
\hline$p$ & $\frac{1}{12}+\frac{5}{12}(D+F)^{2}$ & $-\frac{1}{6}-\frac{5}{6}\left(\frac{D^{2}}{3}+F^{2}\right)$ \\
$n$ & $-\frac{1}{12}-\frac{5}{6}(D+F)^{2}$ & $\frac{1}{12}+\frac{5}{12}(D-F)^{2}$ \\
$\Xi^{-}$ & $-\frac{1}{12}-\frac{5}{12}(D-F)^{2}$ & $-\frac{1}{6}-\frac{1}{6}\left(\frac{2}{3} D^{2}+F^{2}\right)$ \\
$\Xi^{0}$ & $\frac{1}{12}+\frac{5}{12}(D-F)^{2}$ & $-\frac{1}{12}-\frac{5}{12}(D+F)^{2}$ \\
$\Sigma^{+}$ & $\frac{1}{6}+\frac{5}{6}\left(\frac{2}{3} D^{2}+F^{2}\right)$ & $\frac{1}{12}+\frac{5}{12}(D+F)^{2}$ \\
$\Sigma^{-}$ & $-\frac{1}{6}-\frac{5}{6}\left(\frac{2}{3} D^{2}+F^{2}\right)$ & $-\frac{1}{12}-\frac{5}{12}(D-F)^{2}$ \\
$\Lambda$ & 0 & $-\frac{5}{6} D F$ \\
$\Sigma^{0} \Lambda$ & $\frac{5}{3 \sqrt{3}} D F$ & $\frac{5}{6 \sqrt{3}} D F$ \\
\hline
\end{tabular}

\begin{tabular}{|l|c|c|}
\hline & \multicolumn{2}{|c|}{$\beta_{B}^{\prime(X)}$} \\
\hline & $\pi$ & $K$ \\
\hline$p$ & $-\frac{5}{27} \mathcal{C}^{2}$ & $\frac{5}{108} \mathcal{C}^{2}$ \\
$n$ & $\frac{5}{27} \mathcal{C}$ & $\frac{5}{54} \mathcal{C}^{2}$ \\
$\Xi^{-}$ & $-\frac{5}{54} \mathcal{C}^{2}$ & $-\frac{5}{108} \mathcal{C}^{2}$ \\
$\Xi^{0}$ & $\frac{5}{54} \mathcal{C}^{2}$ & $\frac{5}{27} \mathcal{C}^{2}$ \\
$\Sigma^{+}$ & $\frac{5}{108} \mathcal{C}^{2}$ & $-\frac{5}{27} \mathcal{C}^{2}$ \\
$\Sigma^{-}$ & $-\frac{5}{108} \mathcal{C}^{2}$ & $-\frac{5}{54} \mathcal{C}^{2}$ \\
$\Lambda$ & 0 & $\frac{5}{36} \mathcal{C}^{2}$ \\
$\Sigma^{0} \Lambda$ & $-\frac{5}{3 \sqrt{3}} \mathcal{C}^{2}$ & $-\frac{5}{36 \sqrt{3}} \mathcal{C}^{2}$ \\
\hline
\end{tabular}




\section{FIGURES}

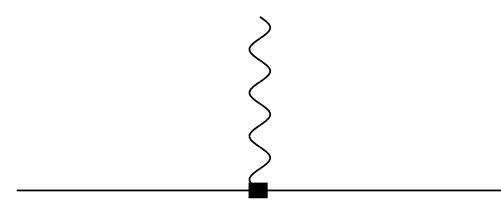

(a)

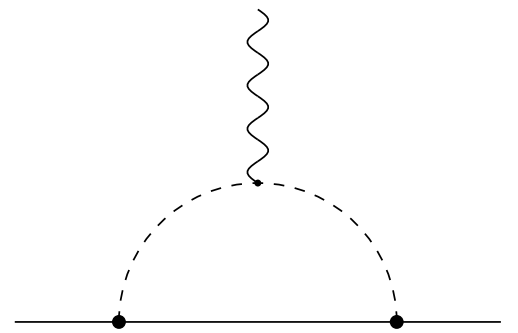

(c)

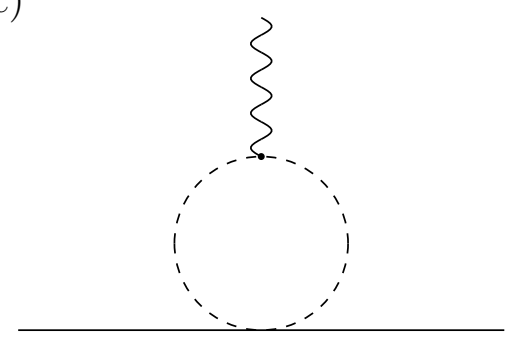

(e)

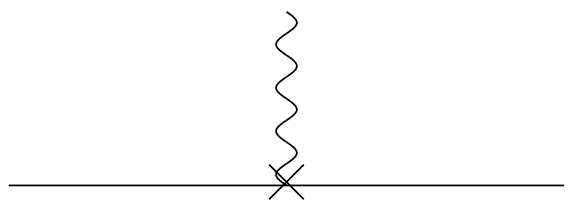

(b)

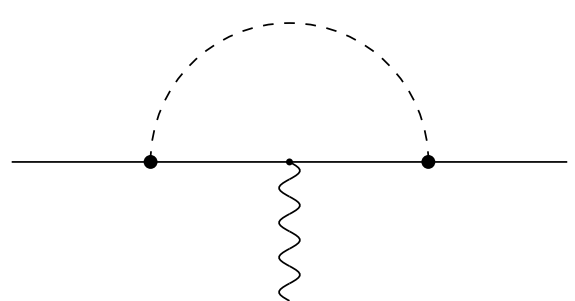

(d)

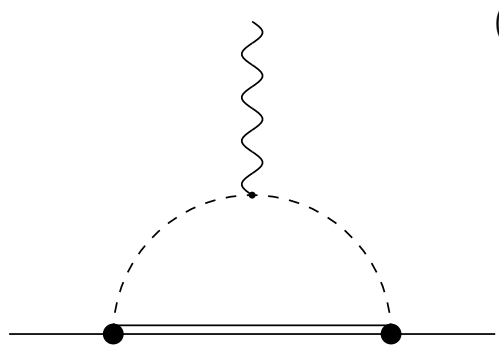

(f)

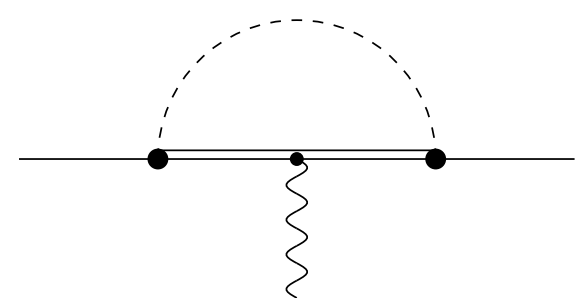

$(\mathrm{g})$

FIG. 1. Loop diagrams contributing to charge radius at $\mathcal{O}\left(1 / \mathrm{HEAVY}^{2}\right)$. The single internal line denotes an intermediate octet state while the double line denotes a decuplet state. 


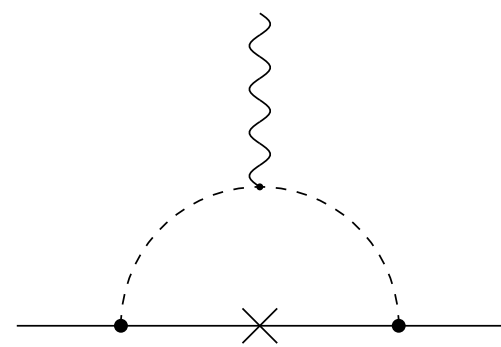

(a)

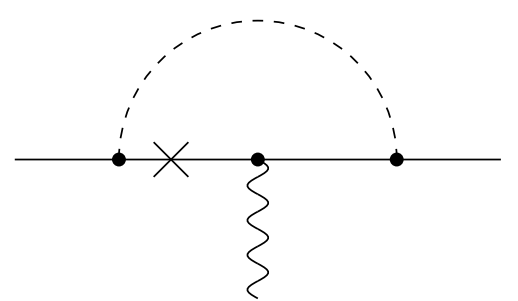

(c)

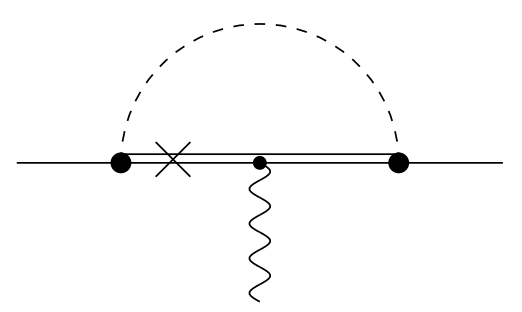

(e)

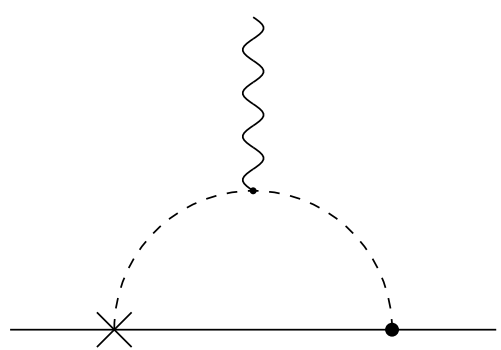

(g)

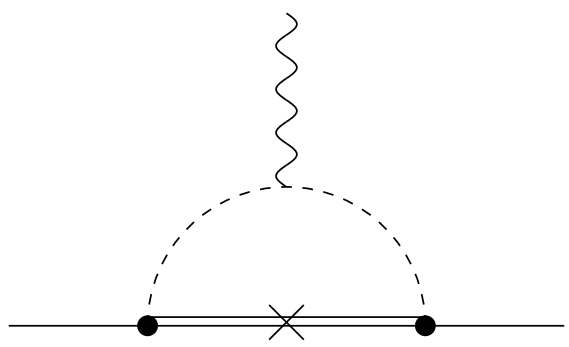

(b)

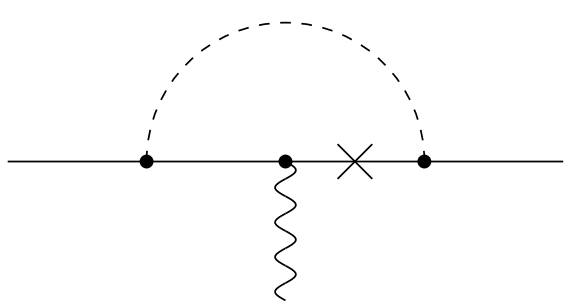

(d)

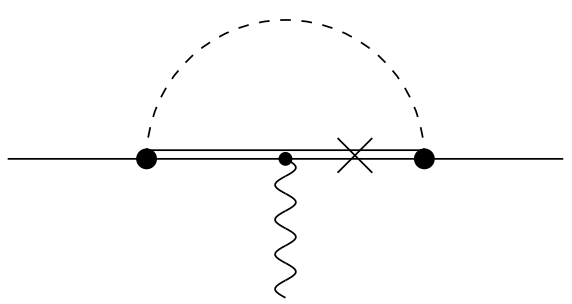

(f)

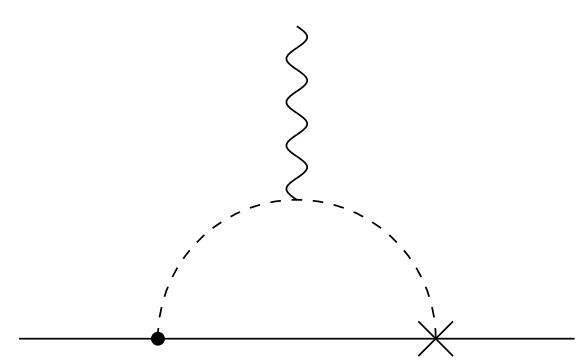

(h)

FIG. 2. Loop diagrams contributing to the charge radius at $\mathcal{O}\left(1 / \mathrm{HEAVY}^{3}\right)$. The " $\times$ " denotes $\mathcal{O}\left(1 / M_{N}\right)$ vertex. 